\title{
An evaluation of the clinical utility of C-reactive protein and antibiotic use in patients undergoing major head and neck reconstructive surgery with outcome assessment
}

\author{
Natalie Archer ${ }^{1}(\mathbb{D}) \cdot$ Lara Zebic $^{1} \cdot$ Natalie Turton ${ }^{1} \cdot$ James Higginson $^{1}{ }^{1} \cdot$. Matthew Idle ${ }^{1} \cdot$ Prav Praveen $^{1}$. \\ Timothy Martin ${ }^{1} \cdot$ Sat Parmar $^{1} \cdot$ Omar Breik $^{1}$
}

Received: 19 January 2021 / Accepted: 2 September 2021 / Published online: 2 October 2021

(c) The Author(s), under exclusive licence to Springer-Verlag GmbH Germany, part of Springer Nature 2021

\begin{abstract}
Purpose This ambispective observational study aims to evaluate the local utility of peri-operative CRP testing and prophylactic antibiotics in relation to post-operative complications in patients who have undergone major head and neck oncological reconstructive surgery.

Results A total of 79 patients were identified for inclusion; CRP testing was undertaken within the first 3 days postoperatively in 78/79 cases. Results demonstrated no benefit of extended prophylactic antibiotic use in reducing post-operative infection. Forty-two post-operative complications arose. In the prospective arm, CRP did not influence the decision to commence antibiotic therapy for any of the surgical site infections. Age, diabetes, smoking, or high body mass index (BMI) did not appear to affect the incidence of postoperative infection $(p>0.05)$. There is no evidence that more than $24 \mathrm{~h}$ of antibiotic prophylaxis is indicated for patients undergoing head and neck reconstructive surgery.

Conclusion Everyone who is involved in peri-operative patient care should be educated regarding the appropriate use of CRP testing, with the implementation of protocols required to standardize CRP testing and prophylactic antibiotic prescription.
\end{abstract}

Keywords CRP $\cdot$ Post-operative $\cdot$ Complications $\cdot$ Antibiotic $\cdot$ Head and neck $\cdot$ Oncology

Natalie Archer

natalie.archer3@nhs.net

Lara Zebic

lara.zebic@nhs.net

Natalie Turton

natalieturton93@gmail.com

James Higginson

james.higginson@cantab.net

Matthew Idle

matthew.idle@uhb.nhs.uk

Prav Praveen

prav.praveen@uhb.nhs.uk

Timothy Martin

timothy.martin@uhb.nhs.uk

Sat Parmar

sat.parmar@uhb.nhs.uk

Omar Breik

omar.breik@gmail.com

1 Department of Oral and Maxillofacial Surgery, Queen Elizabeth Hospital, Birmingham B15 2GW, UK

\section{Introduction}

Following major head and neck oncological reconstructive surgery, infective complications of the lower respiratory tract, urinary tract, or surgical wounds are common. The incidence of wound infection following major head and neck cancer surgery has been reported between 9.1 and $87 \%[1,2]$ with significant implications for surgical outcomes.

It is imperative to monitor for evidence of post-operative infection, with the effective use of available special and haematological investigations, to establish a source and enable rapid and judicious management, whilst maintaining effective antibiotic stewardship. Prophylactic post-operative antibiotics are sometimes employed in an attempt to reduce this risk; however, optimal regimes continue to be debated amongst experts and robust evidence for their extended use is lacking.

C-reactive protein (CRP) is a haematological marker that is commonly used to identify post-operative infection. It is synthesised and secreted by the liver in response to systemic inflammation [3]. In the post-operative setting, it 
is proposed to guide an objective, rational use of antibiotics [4], with some studies suggesting that antibiotic initiation and treatment duration is reduced when CRP is used [5]. However, it is important to consider that CRP is also affected by comorbidities, non-infective inflammation, and trauma, including surgery itself $[3,6]$. CRP is expected to rise in the first 3 days post-operatively; therefore, its use in this time period is limited and not recommended [7].

Limited evidence exists on the peri-operative use of CRP testing within head and neck microvascular reconstructive surgery and its influence on antibiotic prescribing for postoperative complications. The aim of this observational project was to determine our current practice by evaluating the clinical utility of CRP for post-operative monitoring, and antibiotic use in patients that have undergone major head and neck reconstructive surgery in our unit.

\section{Materials and methods}

This ambispective service evaluation was undertaken at the Queen Elizabeth Hospital, Birmingham, UK. All consecutive patients that had undergone primary major head and neck reconstructive oncological surgery between 1 January 2019 and 30 August 2019 were identified and case-records were analysed retrospectively; all cases between 1 September 2019 and 31 March 2020 were evaluated prospectively, without any alteration to the nature of patients' treatment. The study was halted prematurely due to the COVID-19 pandemic. The ambispective design was chosen, because it was felt that prospective data collection would not only allow more accurate recording of complications, but will also allow accurate recording of factors affecting decisionmaking. The consultant clinicians were not aware that their decision-making was being observed to eliminate the Hawthorn effect [8]. No results from the retrospective component were shared prior to commencing the prospective arm.

Data was collected using a standardised electronic form on Microsoft Excel. The age, comorbidities, and operation completed in each case were recorded, in addition to the post-operative prophylactic antibiotic regime prescribed. The initiation and duration of subsequent antibiotics prescribed throughout the inpatient stay were also identified from electronic, time-stamped drug charts. In parallel to this, information regarding any post-operative complications was recorded, including the clinical features and investigations undertaken. Post-operative complications were categorised into infective and non-infective complications, with infective complications further broken-down dependent on infection site. Surgical site infections were defined according to the Clavien-Dindo classification [9].

The frequency and timing of CRP testing was identified from electronic biochemistry flowsheet and analysed in relation to complications recorded and antibiotic prescribing. The blood samples were processed at the Department of Medical Biochemistry and Immunology at University Hospital Birmingham using a turbidometric immunoprecipitation assay on an Abbott Aeroset c8000 analyzer.

All descriptive and analytical statistical operations were performed using the $\mathrm{R}$ statistical language version 4.0.1 (x). Categorical data were analysed using Fisher's exact test, parametric data were analysed using a 2-tailed $t$ test, and non-parametric data were analysed using the Wilcoxon test.

\section{Results}

\section{Patient demographics}

A total of 79 patient-cases were identified for inclusion. Of these, $40(50.6 \%)$ cases were analysed retrospectively and $39(49.4 \%)$ were analysed prospectively. The median age was 65 (range 22-84); 36 patients were female and 43 were male (Table 1). Patient comorbidities are summarised in Table 1, with the most prevalent being hypertension $(n=29)$. Patients underwent a variety of reconstructive surgeries and all except two had a tracheostomy placed intra-operatively. The mean length of inpatient stay was 17.2 days.

\section{Post-operative prophylactic antibiotic regimes}

Patients were prescribed prophylactic broad-spectrum postoperative antibiotics. There was heterogeneity in the duration of the prophylactic antibiotics, with a median duration of 3 days, and a range of $1-9$. The three most common regimes were $24 \mathrm{~h}(n=23,29.1 \%), 48 \mathrm{~h}(n=16,20.3 \%)$, and 5 days $(n=33,41.8 \%)$ (Fig. 1). When patients were classified according to post-operative antibiotic regime $(24 \mathrm{~h}, 48 \mathrm{~h}$, or 5 days), no statistically significant difference between the duration of post-operative antibiotics and the incidence of post-operative infection was identified $(p=0.357)$.

\section{Post-operative complications}

A large proportion of patients experienced post-operative complications $(34 / 79,43 \%)$, with a total of 42 complications recorded and 9 patients experiencing multiple complications (Fig. 2). Of these, 38/42 (90.5\%) were infective and $4 / 42(9.5 \%)$ were non-infective complications. Infective complications were divided into surgical site infection $18 / 38$ (47.4\%); nosocomial infection 14/38 (36.8\%), such as chest or urine; and infection of unknown origin $6 / 38(15.8 \%)$. Surgical site infections were classified by the Clavien-Dindo classification and were further categorised by location; 
Table 1 Patient demographics

\begin{tabular}{|c|c|c|c|c|}
\hline & & $\begin{array}{l}\text { Retrospective arm } \\
n=40\end{array}$ & $\begin{array}{l}\text { Prospective arm } \\
n=39\end{array}$ & $\begin{array}{l}\text { Overall } \\
N=79\end{array}$ \\
\hline \multicolumn{5}{|l|}{ Gender } \\
\hline \multicolumn{2}{|l|}{ Male } & 22 & 21 & 43 \\
\hline \multicolumn{2}{|l|}{ Female } & 18 & 18 & 36 \\
\hline \multicolumn{5}{|l|}{ Age } \\
\hline \multicolumn{2}{|l|}{ Median } & 65 & 65 & 65 \\
\hline \multicolumn{2}{|l|}{ Range } & $27-84$ & $22-84$ & $22-84$ \\
\hline \multicolumn{5}{|l|}{ Comorbidities } \\
\hline \multirow[t]{4}{*}{ Cardiovascular } & Myocardial infarct & 2 & 2 & 4 \\
\hline & Arrhythmia & 3 & 3 & 6 \\
\hline & Hypertension & 21 & 8 & 29 \\
\hline & Angina/coronary artery disease & 4 & 3 & 7 \\
\hline \multirow[t]{2}{*}{ Respiratory } & Asthma & 2 & 3 & 5 \\
\hline & COPD & 1 & 2 & 3 \\
\hline \multirow[t]{3}{*}{ Gastrointestinal } & Alcoholic liver cirrhosis & 1 & 1 & 2 \\
\hline & Inflammatory bowel disease & 0 & 1 & 1 \\
\hline & Gastro-oesophageal reflux & 0 & 2 & 2 \\
\hline \multirow[t]{2}{*}{ Endocrine } & Diabetes mellitus & 2 & 6 & 8 \\
\hline & Hypothyroidism & 7 & 2 & 9 \\
\hline Renal & Chronic kidney disease & 0 & 1 & 1 \\
\hline \multirow[t]{2}{*}{ Neurological } & Stroke/TIA & 0 & 1 & 1 \\
\hline & Epilepsy & 1 & 0 & 1 \\
\hline \multirow[t]{2}{*}{ Rheumatologic } & Sjogren's syndrome & 2 & 0 & 2 \\
\hline & Raynaud's syndrome & 1 & 0 & 1 \\
\hline Coagulopathy & Coagulation disorder & 1 & 0 & 1 \\
\hline \multirow[t]{3}{*}{ Body weight } & $\mathrm{BMI}>30$ & 11 & 7 & 18 \\
\hline & Median & 25.7 & 25.5 & 25.5 \\
\hline & Range & $17.9-50.2$ & $16.7-37.2$ & $16.7-50.2$ \\
\hline Substance abuse & Alcohol & 2 & 1 & 3 \\
\hline \multirow[t]{2}{*}{ Smoking history } & Never smoked & 25 & 18 & 43 \\
\hline & Current/previous smoker & 15 & 21 & 36 \\
\hline \multicolumn{5}{|c|}{ Operation details } \\
\hline \multicolumn{2}{|l|}{ Tracheostomy } & 39 & 38 & 77 \\
\hline \multirow[t]{2}{*}{ Neck dissection } & Unilateral & 31 & 27 & 58 \\
\hline & Bilateral & 9 & 12 & 21 \\
\hline \multirow[t]{3}{*}{ Resection } & Soft tissue & 17 & 23 & 40 \\
\hline & Hard tissue & 18 & 11 & 29 \\
\hline & Both & 5 & 5 & 10 \\
\hline \multirow[t]{7}{*}{ Reconstruction } & Radial forearm & 17 & 17 & 34 \\
\hline & Fibula & 10 & 5 & 15 \\
\hline & Anterolateral thigh & 4 & 14 & 18 \\
\hline & Scapula & 4 & 3 & 7 \\
\hline & Pectoralis major & 2 & 0 & 2 \\
\hline & Deep inferior circumflex iliac artery & 2 & 0 & 2 \\
\hline & Jejunum & 1 & 0 & 1 \\
\hline
\end{tabular}

$10 / 18(55.6 \%)$ at the neck dissection site, $6 / 18(33.3 \%)$ at the donor site, and $2 / 18(11.1 \%)$ at the recipient site.

Surgical site infections arose at a mean of 13.4 (range 4-31) days post-operatively. Most complications were
Clavien-Dindo grade 2 (managed with pharmacological intervention alone-Table 2) [9]. 
Fig. 1 Duration of post-operative prophylactic antibiotics and the proportion of patients who experienced post-operative infection
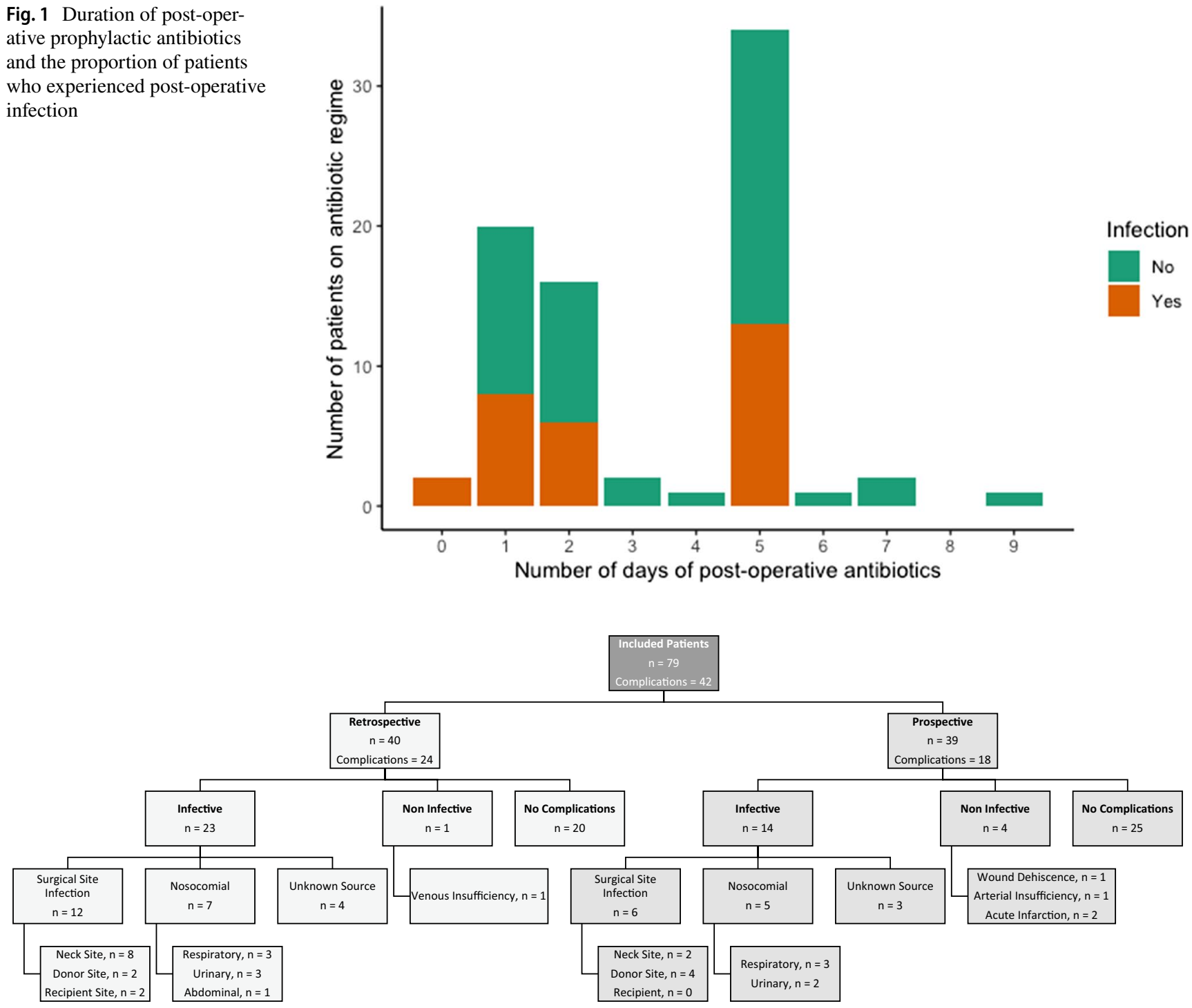

Fig. 2 Post-operative complications encountered

Table 2 Clavien-Dindo classification of post-operative complications

\begin{tabular}{ll}
\hline Clavien-Dindo classification (grade) & $\begin{array}{l}\text { Number of } \\
\text { complica- } \\
\text { tions }\end{array}$ \\
\hline 2 & 37 \\
$3 \mathrm{~b}$ & 2 \\
$4 \mathrm{a}$ & 3 \\
5 & 1 \\
\hline
\end{tabular}

Age, diabetes, smoking, or high body mass index (BMI) did not appear to affect the incidence of post-operative infection $(p>0.05)$.
The inpatient stay for those who developed post-operative complications increased from a median of 11 , for those without complications, to 17 days.

\section{CRP utility}

A total of 617 CRP tests were undertaken, with a median of 7 per patient. In $46 / 79(58.2 \%)$ cases, CRP was taken preoperatively, and 78/79 had CRP taken within the first 3 days after surgery. In 14/79 (17.7\%) cases, CRP testing was undertaken on all three consecutive days post-operatively (Fig. 3). The median number of CRP tests undertaken in those without any recorded complication was 5.5, compared to 9 in those who experienced complications.

In $13 / 16(81.3 \%)$ cases, CRP tests were taken on the day of surgical site infection diagnosis, with levels elevated in 
Fig. 3 Number of CRP tests undertaken post-operatively

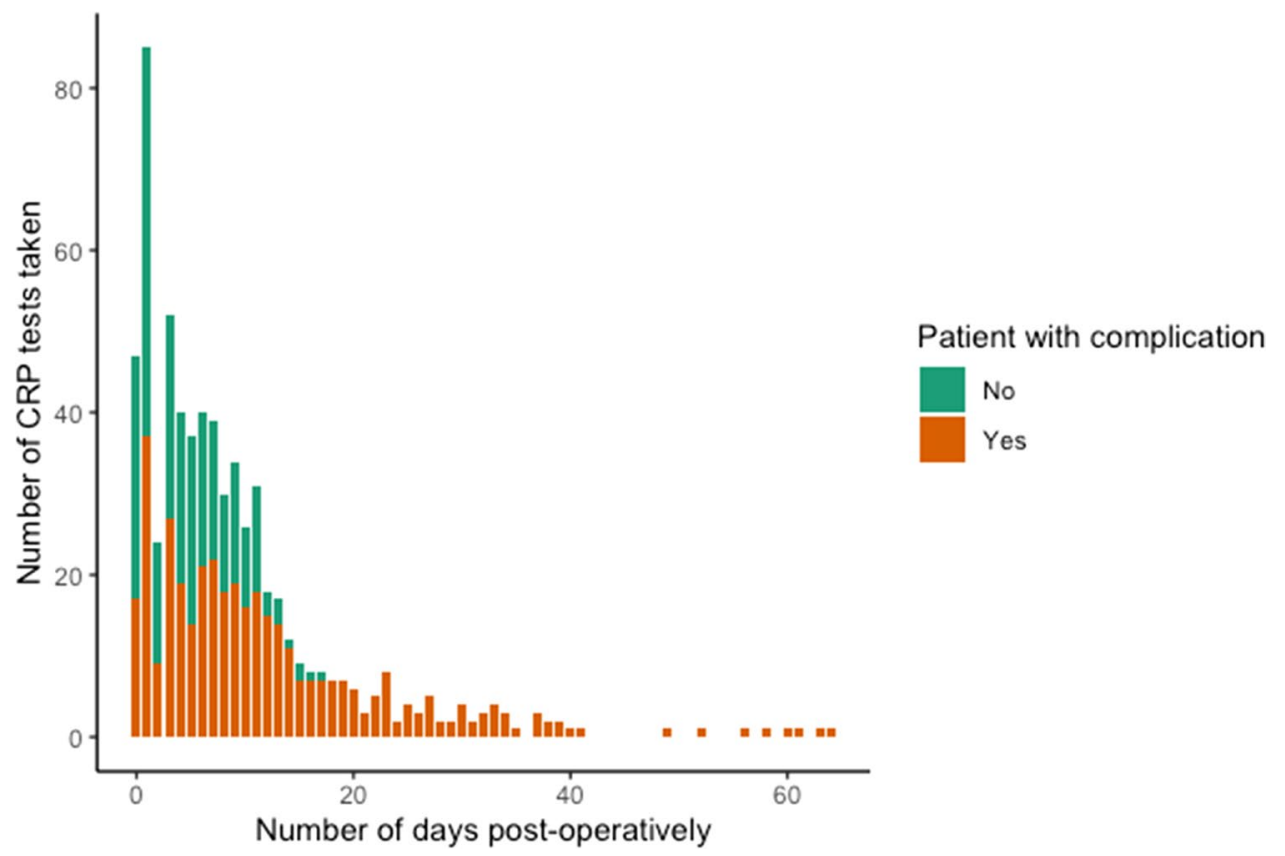

4/13 (30.8\%) patients. In 5/13 (38.5\%) cases, CRP only became elevated between 2 and 4 days after diagnosis. In the prospective arm, CRP did not influence the decision to commence antibiotic therapy for any of the surgical site infections. Notably, in one case, CRP was elevated 4 days prior to clinical suppuration; however, antibiotics were not commenced until clinical signs were seen.

Overall, 14/19 (73.7\%) of nosocomial and unknown infections had CRP taken at the time of diagnosis. Of these, 6/14 (42.9\%) CRP values were elevated, 2 of which aided antibiotic commencement. Non-infective complications were not associated with increased CRP levels.

\section{Discussion}

In this ambispective study, we identified that CRP has limited predictive value for post-operative infective complications in head and neck reconstructive surgery and extended antibiotic prophylactic regimens had no clinical benefit in reducing post-operative infections.

\section{Current practice}

The lack of definitive protocols within the investigating unit has led to a wide variation in post-operative antibiotic use amongst head and neck surgeons. With the growing concerns about antibiotic stewardship, more evidencebased prescribing is needed. There are still high rates of inappropriate prophylactic antibiotics used in surgical settings. In addition to improving patient safety, these measures will help combat antibiotic resistance and reduce costs [10]. A recent meta-analysis by Vila et al. [11] concluded that there is no benefit to extending prophylactic antibiotic regimes from 1 to 5 days. Yet, another recent meta-analysis by Haidar et al. [12] suggests that whilst $24 \mathrm{~h}$ of antibiotics may be sufficient, it is difficult to draw definitive conclusions and highlights a need for further prospective trials. A balanced perspective is given by the British Association of Head and Neck Oncologists guidelines which advise that antibiotic regimes longer than $24 \mathrm{~h}$ have no additional benefit [13], which is coincident with recommendations for prophylactic antibiotics in operative facial fracture cases [14]. Our study provides further evidence that corroborates these guidelines and demonstrates that there is no advantage of prophylactic antibiotics for greater than $24 \mathrm{~h}$ in patients undergoing head and neck reconstructive surgery.

The overall rate of complication in our study was $43 \%$. Surgical site infection is documented in the literature as 9.1-87\% [1, 2]. Our results revealed a surgical site complication rate of $18.8 \%$. The risk of developing postoperative complications has a substantial impact on the post-operative healing process and prolonged hospitalisation for further investigations and treatment [15]. Factors which have been associated with an increased risk of postoperative complications in other studies include diabetes, higher BMI, tobacco use, hypothyroidism, and tracheostomy placement [16-18]. However, in our cohort diabetes, higher BMI and tobacco use had no significant association with the incidence of post-operative infection. Due to our sample size, we were unable to evaluate the incidence of 
complications relating to hypothyroidism and tracheostomy placement.

\section{CRP utility}

National Institute of Clinical Excellence (NICE) guidance does not include CRP testing as part of their recommended routine pre-operative haematological investigations [19], yet over half of the patients in this cohort had CRP requested pre-operatively. Furthermore, despite an established evidence base demonstrating a rise in CRP peaks at $48 \mathrm{~h}$ following surgical trauma, 78/79 (98.7\%) patients had at least one CRP test taken within the first 3 days post-operatively. Following surgery CRP levels may elevate up to 100 times their basal concentrations in healthy individuals, before gradually declining in the absence of infection [20]. Therefore, with no clinical benefit, it can be argued that CRP testing in this period is unjustified. CRP is the most inappropriately ordered blood test; with a CRP test costing on average $£ 1.03$, this can amount to a significant additional healthcare cost when considering multi-specialties and sites [21].

There is little evidence as to whether elevated CRP affects peri-operative clinical decisions in regard to managing postoperative infection or complications in practice [7]. When evaluating patients presenting with infection, the CRP often peaks 3 days after the appearance of infective symptoms; hence, it cannot be used as a diagnostic marker in isolation, rather should be used in conjunction with clinical signs and special investigations [22]. In the setting of patients with known malignancy, and after undergoing major reconstructive surgery, the clinical value of CRP is even lower due to the lack of specificity and its delayed response. A rise in CRP was noted in less than half of the cases with infective complications. Additionally, this elevation most commonly occurred 2-4 days after the development of clinical signs which meant CRP did not influence clinical decision-making. CRP did not reliably predict post-operative infective or non-infective complications.

There is reason to suggest that CRP is more valuable in monitoring response to treatment as opposed to establishing an infective diagnosis [23]. Although this was not evaluated in this study, the role of CRP in post-operative care should be limited to monitoring response to treatment of infection $[24,25]$. To improve the clinical utility of CRP, prevent unnecessary testing, and therefore improve the use of resources, it is essential to identify clear justification for testing.

Our study has provided an insightful evaluation of current practice at a single unit, and highlighted areas for local improvement. Our findings have prompted the development of the following protocol in our unit: (1) Prophylactic postoperative antibiotics are to be administered for only $24 \mathrm{~h}$ after major head and neck reconstructive surgery. (2) CRP will not be tested pre-operatively, or within the first 3 days post-operatively. (3) CRP will only be requested if there is clinical suspicion of infection for monitoring response to treatment. As well as the surgical team, these changes have been disseminated to the pre-admission and critical care teams who are involved in the request of haematological investigations in the immediate peri-operative period. However, it is difficult to draw more general conclusions from the small heterogeneous cohort presented. Larger prospective studies are required which may also consider alternative haematological markers such as neutrophil counts.

\section{Conclusions}

CRP is expected to rise post-operatively, with a peak on day 3 as part of the normal inflammatory response to surgery. There is no indication to routinely request CRP levels during this period, or at pre-assessment. CRP was not reliable as a predictive marker of post-operative complications. All teams who are involved in peri-operative patient care should be educated regarding the judicious use of CRP testing.

Within this cohort, there was no evidence to suggest that more than $24 \mathrm{~h}$ of antibiotic prophylaxis is indicated for patients undergoing head and neck reconstructive surgery.

Data availability Data are available within the article or in its supplementary materials.

Code availability Not applicable.

\section{Declarations}

Ethics approval Not applicable, observational study with no alterations made to patient treatment.

Consent to participate Not applicable.

Consent for publication Not applicable.

Conflict of interest The authors declare no competing interests.

\section{References}

1. Simo R, French G (2006) The use of prophylactic antibiotics in head and neck oncological surgery. Curr Opin Otolaryngol Head Neck Surg 14:55-61. https://doi.org/10.1097/01.moo.00001 93183.30687.d5

2. Cannon RB, Houlton JJ, Mendez E, Futran ND (2017) Methods to reduce postoperative surgical site infections after head and neck oncology surgery. Lancet Oncol 18:e405-e413. https://doi.org/10. 1016/S1470-2045(17)30375-3 
3. Vigushin DM, Pepys MB, Hawkins PN (1993) Metabolic and scintigraphic studies of radioiodinated human C-reactive protein in health and disease. J Clin Invest 91:1351-1357. https://doi.org/ 10.1172/JCI116336

4. Fontela PS, O'Donnell S, Papenburg J (2018) Can biomarkers improve the rational use of antibiotics? Curr Opin Infect Dis 31:347-352. https://doi.org/10.1097/QCO.0000000000000467

5. Petel D, Winters N, Gore GC et al (2018) Use of C-reactive protein to tailor antibiotic use: a systematic review and metaanalysis. BMJ Open 8:e022133. https://doi.org/10.1136/bmjop en-2018-022133

6. Black S, Kushner I, Samols D (2004) C-reactive protein. J Biol Chem 279:48487-48490. https://doi.org/10.1074/jbc.R400025200

7. Cole DS, Watts A, Scott-Coombes D, Avades T (2008) Clinical utility of peri-operative C-reactive protein testing in general surgery. Ann R Coll Surg Engl 90:317-321. https://doi.org/10.1308/ $003588408 X 285865$

8. McCambridge J, Witton J, Elbourne DR (2014) Systematic review of the Hawthorne effect: new concepts are needed to study research participation effects. J Clin Epidemiol 67:267-277

9. Clavien PA, Barkun J, de Oliveira ML et al (2009) The Clavien-Dindo classification of surgical complications. Ann Surg 250:187-196. https://doi.org/10.1097/SLA.0b013e3181b13ca2

10. Chiesa-Estomba CM, Ninchritz E, González-García JA et al (2019) Antibiotic prophylaxis in clean head and neck surgery: an observational retrospective single-centre study. Ear Nose Throat J 98:362-365. https://doi.org/10.1177/0145561319853520

11. Vila PM, Zenga J, Fowler S, Jackson RS (2017) Antibiotic prophylaxis in clean-contaminated head and neck surgery: a systematic review and meta-analysis. Otolaryngol Neck Surg 157:580-588. https://doi.org/10.1177/0194599817712215

12. Haidar YM, Tripathi PB, Tjoa T et al (2018) Antibiotic prophylaxis in clean-contaminated head and neck cases with microvascular free flap reconstruction: a systematic review and meta-analysis. Head Neck 40:417-427. https://doi.org/10.1002/hed.24988

13. BAHNO (2016) UK Head and Neck Cancer guidelines. In: Br. Assoc. Head Neck Oncol. https://www.bahno.org.uk/_userfiles/ pages/files/ukheadandcancerguidelines2016.pdf. Accessed 18 Jan 2021

14. Forrester JD, Wolff CJ, Choi J et al (2020) Surgical infection society guidelines for antibiotic use in patients with traumatic facial fractures. Surg Infect (Larchmt) sur.2020.107. https://doi.org/10. 1089/sur.2020.107

15. Pool C, Kass J, Spivack J et al (2016) Increased surgical site infection rates following clindamycin use in head and neck free tissue transfer. Otolaryngol Neck Surg 154:272-278. https://doi.org/10. $1177 / 0194599815617129$
16. Bartella AK, Lemmen S, Burnic A et al (2018) Influence of a strictly perioperative antibiotic prophylaxis vs a prolonged postoperative prophylaxis on surgical site infections in maxillofacial surgery. Infection 46:225-230. https://doi.org/10.1007/ s15010-017-1110-4

17. Mitchell RM, Mendez E, Schmitt NC et al (2015) Antibiotic prophylaxis in patients undergoing head and neck free flap reconstruction. JAMA Otolaryngol Neck Surg 141:1096. https://doi. org/10.1001/jamaoto.2015.0513

18. Balamohan SM, Sawhney R, Lang DM et al (2019) Prophylactic antibiotics in head and neck free flap surgery: a novel protocol put to the test. Am J Otolaryngol 40:102276. https://doi.org/10.1016/j. amjoto.2019.102276

19. NICE (2016) The use of pre-operative tests for elective surgery: evidence, methods and guidance. https://www.nice.org.uk/guida nce/ng45/resources/routine-preoperative-tests-for-elective-surge ry-pdf-1837454508997. Accessed 18 Jan 2021

20. Gambino R (1997) C-reactive protein-undervalued, underutilized. Clin Chem 43:2017-2018. https://doi.org/10.1093/clinc hem/43.11.2017

21. Akhtar W, Chung Y (2014) Saving the NHS one blood test at a time. BMJ Qual Improv Rep 2:u204012.w1749. https://doi.org/ 10.1136/bmjquality.u204012.w1749

22. Póvoa P (2002) C-reactive protein: a valuable marker of sepsis. Intensive Care Med 28:235-243. https://doi.org/10.1007/ s00134-002-1209-6

23. Khan MH, Smith PN, Rao N, Donaldson WF (2006) Serum C-reactive protein levels correlate with clinical response in patients treated with antibiotics for wound infections after spinal surgery. Spine J 6:311-315. https://doi.org/10.1016/j.spinee.2005. 07.006

24. Póvoa P, Almeida E, Moreira P et al (1998) C-reactive protein as an indicator of sepsis. Intensive Care Med 24:1052-1056. https:// doi.org/10.1007/s001340050715

25. Adnet F, Borron SW, Vicaut E et al (1997) Value of C-reactive protein in the detection of bacterial contamination at the time of presentation in drug-induced aspiration pneumonia. Chest 112:466-471. https://doi.org/10.1378/chest.112.2.466

Publisher's note Springer Nature remains neutral with regard to jurisdictional claims in published maps and institutional affiliations. 